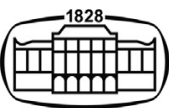

AKADÉMIAI KIADÓ

\title{
Impact of COVID-19 on gaming disorder: Monitoring and prevention
}

\author{
CHIH-HUNG $\mathrm{KO}^{1,2,3 *}$ (๑) and JU-YU YEN ${ }^{1,4,5}$
}

\section{Journal of Behavioral Addictions}

9 (2020) 2, 187-189

DOI:

10.1556/2006.2020.00040

(c) 2020 The Author

\section{LETTER TO THE EDITOR}



*Corresponding author. Department of Psychiatry, Kaohsiung Municipal Siaogang Hospital, 100 Tzyou 1st Road, Kaohsiung, 80708, Taiwan, ROC

E-mail: chihhungko@gmail.com
${ }^{1}$ Department of Psychiatry, Kaohsiung Medical University Hospital, Kaohsiung Medical University, 100 Tzyou 1st Rd, Kaohsiung City, 807, Taiwan, ROC

2 Department of Psychiatry, Kaohsiung Municipal Siaogang Hospital, Kaohsiung Medical University, 482 San-Ming Rd, Kaohsiung City, 812, Taiwan, ROC

${ }^{3}$ Research Center for Substance and Behavior Addiction, Kaohsiung Medical University, Kaohsiung, Taiwan, ROC

${ }^{4}$ Department of Psychiatry, Faculty of Medicine, College of Medicine, Kaohsiung Medical University, 100 Shi-Chuan 1st Rd, Kaohsiung City, 807, Taiwan, ROC

${ }^{5}$ Department of Psychiatryh, Kaohsiung Municipal Ta-Tung Hospital, Kaohsiung Medical University, Kaohsiung City, 812, Taiwan, ROC

Received: March 28, 2020 • Revised manuscript received: May 1, 2020; May 5, 2020; May 24, 2020 • Accepted: May 24, 2020 • Published online: June 6, 2020

\begin{abstract}
The global coronavirus disease 2019 (COVID-19) outbreak has necessitated physical distancing, lockdown, contact tracing, and self-quarantine so as to prevent the spread of the disease. Amid the outbreak, gaming data usage has reportedly increased in the United States, and game download volume has reached a record high in Europe. Because gaming can be used to cope with the psychological stress from the outbreak, therefore mental health professionals should be aware of how increased gaming during the pandemic may contribute to risk of gaming disorder, especially if the pandemic persists. Mental health professionals should thus formulate safe social interaction alternatives for people, particularly adolescents, who have gaming disorder risk.
\end{abstract}

\section{KEYWORDS}

COVID-19, gaming disorder, adolescents, physical distancing, psychological impact

Coronavirus disease 2019 (COVID-19) originated in Wuhan City, Hubei Province, China. As of May 4, 2020, COVID-19 has become a global pandemic, affecting 214 countries, territories, or areas, with 3,435,894 people infected and 239,604 deaths (World Health Organization [WHO], 2020). Preventive measures, such as wearing a surgical mask, physical distancing, mass testing, contact tracing, and quarantines, have been employed to prevent disease spread (Adhikari et al., 2020). According to Hans Vestberg, CEO of Verizon Communications Inc., gaming increased by $75 \%$ in the United States during the week of March 815,2020 , when many US state governments began adopting pandemic prevention measures (Clifford, 2020). Sensor Tower, an analytics intelligence platform, also reported that European mobile game download volume reached a record high (increasing by 19\%) in March 2020, when strict lockdown measures were implemented (Broughton, 2020).

Alarming information on the outbreak in the media has likely had a profound psychosocial effect on the general population, a situation similar to that which occurred during the 2003 severe acute respiratory syndrome outbreak (Ko, Yen, Yen, \& Yang, 2006). During such times of heightened distress, people require outlets to cope with anxiety and stress. However, popular means of stress relief, such as attending sporting events, celebrations, and religious gatherings, have become prohibited or impracticable due to physical distancing measures. Furthermore, in daily interactions, in-person communication is hindered by wearing of masks, which makes speaking muffled and facial expressions hard to read. Given these limitations, gaming may be employed as a means of escape to alleviate these distresses by many. While gaming may be an adaptive coping strategy on the short term (Russoniello, 
O'Brien, \& Parks, 2009), it can become maladaptive (i.e., a risk of gaming disorder, or other problems) if it develops into the habitualized coping strategy for stress, while other adaptive ones are not applied. It is likely that such a maladaptive use of gaming has become more frequent during the COVID-19 outbreak because many alternative strategies have become impracticable.

School closures and event cancellations particularly have limited social interactions among adolescents during this pandemic. Without following the routine of going to school and participating in other activities, adolescents can become isolated playing video games in their rooms. Furthermore, adolescents are likely to play video games because their other hobbies have become impracticable. Parents may also be preoccupied with COVID-19 prevention, work, and tasks around the house and thus fail to notice excessive gaming in their adolescent children. Adolescents may also stay up late playing video games because they need not go to school the next day. Therefore, GD risk may increase during this pandemic because of the increased opportunity to play video games (at any time) and decreased access to alternative social activities. Furthermore, individuals having GD already are less likely to recover when they have decreased access to alternative avenues for activity, social interaction, and achievement. Thus, adolescents, being especially at risk of developing GD, as well as those already with GD should be appropriately monitored for their gaming patterns, sleep habits, and extent of social isolation in clinical and community settings during the COVID-19 pandemic.

Ensuring absolute compliance with COVID-19 prevention measures, such as school closures, is crucial; however, school administrators must also encourage alternative means of social interaction between students, such as videoconferencing and participation in self-learning programs, as implemented in Junyi Academy in Taiwan. Parents must also make time to interact with their adolescent children as well as monitor and regulate their gaming time, particularly during their regular sleeping hours (Ko, Lin, Lin, \& Yen, 2019).

If outdoor activities and community events involving social interaction are prohibited, at-home activities, such as table games or home exercise, should be designed and provided. Furthermore, rather than playing mainstream video games, educational video games (such as $\mathrm{PaGamO}$, popular in Taiwan) or active games that emphasize exercise or real-world social interaction can be played; such games may be better for the player's mental and physical health than mainstream video game genres such as massively multiplayer online role-playing games, and shooter games (Fu, Burns, Gomes, Savignac, \& Constantino, 2019). The remote conferencing services Cisco Webex and Google Meet can also be used for student-student and student-teacher interactions.

In general, parents and educators must provide alternative avenues for social interaction among adolescents in addition to maintaining their learning motivation and monitoring and regulating their gaming time, all of which could be essential to minimizing GD-related risks during this pandemic (Stavropoulos et al., 2019; Wartberg, Kriston,
\& Kammerl, 2017). Furthermore, mental health professionals must provide emotional support and advice on coping strategies to relieve pandemic-related stress in individuals. They must also pay attention to excessive gaming behavior and its negative consequences, such as sleep disorders or obesity (Ko et al., 2019), among at-risk people, such as adolescents or those with attention deficit/hyperactivity disorder (Yen et al., 2017), depression, or anxiety disorder (Wang et al., 2017).

Funding sources: This study was supported by Taiwan's Ministry of Science and Technology (MOST105-2314-B037-027-MY2, MOST107-2314-B-037-101-MY2), Kaohsiung Municipal Siaogang Hospital (KMHK-104-006; KMHK-103-008), Kaohsiung Medical University Hospital (KMUH105-5R54; KMUH106-6R71), and the Research Center for Substance and Behavior Addiction of Kaohsiung Medical University.

Conflict of interest: Chih-Hung Ko conducted research that was funded directly by Taiwan's Ministry of Science and Technology, Kaohsiung Municipal Siaogang Hospital, Kaohsiung Medical University Hospital, and the Research Center for Substance and Behavior Addiction of Kaohsiung Medical University. Ju-Yu Yen conducted research funded directly by Taiwan's Ministry of Science and Technology, Kaohsiung Municipal Ta-Tung Hospital, and Kaohsiung Medical University Hospital.

\section{REFERENCES}

Adhikari, S. P., Meng, S., Wu, Y. J., Mao, Y. P., Ye, R. X., Wang, Q. Z., \& Zhou, H. (2020). Epidemiology, causes, clinical manifestation and diagnosis, prevention and control of coronavirus disease (COVID-19) during the early outbreak period: A scoping review. Infectious Diseases of Poverty, 9(1), 29. https:// doi.org/10.1186/s40249-020-00646-x.

Broughton, M. (2020, April 17). Europe mobile game revenue hits record high; riot acquires hypixel. The Gaming Economy.

Clifford, T. (2020, May 19). Web traffic spiked 20\% in one week amid coronavirus shutdown, Verizon CEO says. CNBC newsletters.

Fu, Y., Burns, R. D., Gomes, E., Savignac, A., \& Constantino, N. (2019). Trends in sedentary behavior, physical activity, and motivation during a classroom-based active video game program. International Journal of Environmental Research and Public Health, 16(16). https://doi.org/10.3390/ ijerph16162821.

Ko, C. H., Lin, H. C., Lin, P. C., \& Yen, J. Y. (2019). Validity, functional impairment and complications related to Internet gaming disorder in the DSM-5 and gaming disorder in the ICD-11. The Australian and New Zealand journal of Psychiatry, 4867419881499. https://doi.org/10.1177/0004867419881499.

Ko, C. H., Yen, C. F., Yen, J. Y., \& Yang, M. J. (2006). Psychosocial impact among the public of the severe acute respiratory syndrome epidemic in Taiwan. Psychiatry and Clinical 
Neurosciences, 60(4), 397-403. https://doi.org/10.1111/j.1440 1819.2006.01522.x.

Russoniello, C. V., O’Brien, K., \& Parks, J. M. (2009). The effectiveness of casual video games in improving mood and decreasing stress. Journal of Cyber Therapy and Rehabilitation, 2(1), 53+.

Stavropoulos, V., Anderson, E. E., Beard, C., Latifi, M. Q., Kuss, D., \& Griffiths, M. (2019). A preliminary cross-cultural study of Hikikomori and Internet Gaming Disorder: The moderating effects of game-playing time and living with parents. Addictive Behaviors Reports, 9, 001-001. https://doi.org/10.1016/j.abrep.2018.10.001.

Wang, C. Y., Wu, Y. C., Su, C. H., Lin, P. C., Ko, C. H., \& Yen, J. Y. (2017). Association between Internet gaming disorder and generalized anxiety disorder. Journal of Behavioral Addictions, 6(4), 564-571. https://doi.org/10.1556/2006.6.2017.088.
Wartberg, L., Kriston, L., \& Kammerl, R. (2017). Associations of social support, friends only known through the internet, and health-related quality of life with internet gaming disorder in adolescence. Cyberpsychology, Behavior, and Social Networking, 20(7), 436-441. https://doi.org/10.1089/cyber.2016.0535.

World Health Organization. (2020). Coronavirus disease 2019 (COVID-19) situation report 105. Retrieved May 5, 2020, from https://www.who.int/docs/default-source/coronaviruse/situationreports/20200504-covid-19-sitrep-105.pdf?sfvrsn=4cdda8af_2.

Yen, J. Y., Liu, T. L., Wang, P. W., Chen, C. S., Yen, C. F., \& Ko, C. H. (2017). Association between Internet gaming disorder and adult attention deficit and hyperactivity disorder and their correlates: Impulsivity and hostility. Addictive Behaviors, 64, 308-313. https://doi.org/10.1016/j.addbeh.2016.04.024. 